Supporting Information for

\title{
Spectroscopically Resolved Binding Sites for the Adsorption of Sarin Gas in a Metal- Organic Framework: Insights Beyond Lewis Acidity
}

Jacob A. Harvey, ${ }^{\dagger}$ Monica L. McEntee, ${ }^{\ddagger}$ Sergio J. Garibay, ${ }^{\ddagger}$ Erin M. Durke,${ }^{\ddagger}$ Jared B. Decoste,, Jeffery A. Greathouse, ${ }^{\dagger}$ Dorina F. Sava Gallis ${ }^{\S^{*}}$

Geochemistry Department, Sandia National Laboratories, Albuquerque, New Mexico 87125, USA

¥Combat Capabilities Development Command Chemical Biological Center, U.S. Army Research, Development and Engineering Command, 8198 Blackhawk Road, Aberdeen Proving Ground, MD 21010, USA

§ Nanoscale Sciences Department, Sandia National Laboratories, Albuquerque, NM 87185, USA *Corresponding Author: dfsava@ sandia.gov 


\section{Experimental Synthesis and Characterization}

$\mathrm{ZrCl}_{4}(0.135 \mathrm{~g}, 0.81 \mathrm{mmol})$ and terephthalic acid $(0.189 \mathrm{~g}, 0.81 \mathrm{mmol})$ were placed in a Teflon liner and $5 \mathrm{~mL}$ of DMF and $\sim 0.145 \mathrm{~mL}$ of $12 \mathrm{M} \mathrm{HCl} 35 \%$ were added to this mixture. The reaction was placed in an autoclave and heated at $160^{\circ} \mathrm{C}$ for $\sim 24 \mathrm{~h}$. After cooling to room temperature, the crystalline material was recovered by centrifugation and washed with fresh DMF and acetone through centrifugation. The guest exchanged material was heated at $120^{\circ} \mathrm{C}$ under vacuum for BET and TGA analysis.

PXRD was measured on a Rigaku Miniflex 600 diffractometer at $30 \mathrm{kV}, 15 \mathrm{~mA}(\mathrm{CuK} \alpha 1$ radiation, $\lambda=1.54056 \AA$ ) with a scan speed of $5^{\circ} / \mathrm{min}$ and a step size of 0.05 in $2 \theta$ at room temperature and is shown in Figure $\mathrm{S} 1$.

Nitrogen isotherm measurements were carried out on a Micromeritics ASAP 2420 at $77 \mathrm{~K}$ and are shown in Figure S2. Samples were activated at $120^{\circ} \mathrm{C}$ for $16 \mathrm{~h}$ under vacuum on Micromeritics Smart VacPrep instrument and outgas rate below $0.05 \mathrm{mmHg} / \mathrm{min}$ was achieved.

\section{Thermogravimetric analyses}

Samples $\sim 5 \mathrm{mg}$ were analyzed under a stream of air using a TA Instrument Q600 SDT running from room temperature to $600{ }^{\circ} \mathrm{C}$ with a scan rate of $5^{\circ} \mathrm{C} / \mathrm{min}$. TGA under air with $\mathrm{Zr}$-based MOFs generates $\mathrm{ZrO}_{2}$ above $500^{\circ} \mathrm{C}$. After TGA analysis, the weight loss was normalized with respect to $\mathrm{ZrO}_{2}$, where the end plateau was set to $100 \%$. The plateau around $370{ }^{\circ} \mathrm{C}$ corresponds to the dehydroxylated UiO-66 with the formula of $\left[\mathrm{Zr}_{6} \mathrm{O}_{6}(\mathrm{BDC})_{6}\right]$ for an ideal framework. The weight of this structure should be $220 \%$ relative to $\mathrm{ZrO}_{2}$. If missing-linkers defects are present, the framework would appear lighter and this plateau would deviate from the ideal value. Therefore, by measuring this difference, the number of missing linkers per formula unit can be calculated. Using the methods outline by Shearer et al. ${ }^{1}$ it was determined from the TGA data in Figure S3 that the UiO-66 sample used here has $\sim 9 \%$ of its linkers missing exhibiting the formula $\mathrm{Zr}_{6} \mathrm{O}_{6}(\mathrm{BDC})_{5.45}$.

\section{Experimental IR Spectroscopy}

Transmission IR spectroscopy experiments were performed in a vacuum chamber with a base pressure of $\sim 3 \times 10^{-9}$ Torr. A detailed description of the system is explained elsewhere. ${ }^{2}$ Approximately $3 \mathrm{mg}$ of UiO-66 powder was pressed into a 0.004 inch thick W-grid disk, which was attached to copper leads to allow for resistive heating and cooling of the surface in a temperature range from $\sim 85-1000 \mathrm{~K}$ at $0.1 \mathrm{~K}$ resolution. Before GB exposure, the UiO-66 sample was heated up to $400 \mathrm{~K}$ for 45 minutes in vacuum to remove any $\mathrm{H}_{2} \mathrm{O}$ or solvent from the surface. After 45 minutes, the sample was cooled back down to $300 \mathrm{~K}$. An IR spectrum was then taken before GB exposure with an average of 256 scans at a $2 \mathrm{~cm}^{-1}$ resolution. The background spectrum used was the W-grid without MOF pressed into the squares. Minute quantities of GB were introduced into the IR chamber via a variable leak valve. Caution! Experiments using GB should be run by trained personnel using appropriate safety procedures only. Once GB entered the chamber, IR spectra were taken over time at separate intervals. After exposure, the GB feed was closed and the IR chamber was allowed to evacuate, after which an additional IR spectrum was 
obtained to determine if any weakly bound GB molecules desorbed from the surface. Samples were evacuated from what we arbitrarily classify as high $\left(8 \times 10^{-5}\right.$ Torr $)$ and low $\left(5 \times 10^{-6}\right.$ Torr $)$ pressure states. The temperature-programmed desorption-IR (TPD-IR) experiments were performed by heating the GB-exposed sample in vacuum up to $400 \mathrm{~K}$ at $20 \mathrm{~K}$ intervals. Once 400 $\mathrm{K}$ was reached, additional IR spectra were taken at $400 \mathrm{~K}$ to observe any changes to the GB modes on the surface of the MOF.

\section{Computational Details}

The known crystal structure of UiO-66 was used for DFT calculations. ${ }^{3-4}$ In the interest of computational speed, the primitive unit cell was used for all calculations. The unit cell was optimized according to the procedure described below which resulted in cell parameters of $\mathrm{a}=$ $14.67 \AA, b=14.72 \AA, c=14.66 \AA, \alpha=59.9^{\circ}, \beta=59.7^{\circ}$, and $\gamma=59.5^{\circ}$. This compares favorably to the experimental crystal structure parameters of $a, b, c=14.64 \AA$, and $\alpha, \beta, \gamma=60.0^{\circ}$. Initial positions for GB were created by manually placing, with Materials Studio, ${ }^{5}$ the molecule near the intended binding site. The bulk 3D structure of UiO-66, with a missing-linker defect, is depicted in Figure 1 in the manuscript. The binding sites considered in this study are indicated ( $\mathrm{Zr}$ defect, $\mathrm{ZrOH}$, and $\left.\mu_{3}-\mathrm{OH}\right)$.

Calculations of the degradation products on UiO-66 utilized the structures identified in the reaction pathway modeling work done by Troya. ${ }^{6}$ Optimizations were performed using the projector augmented wave approach ${ }^{7-8}$ as implemented in the Vienna Ab Initio Simulation Package. ${ }^{9-12}$ The Perdew-Burker-Ernzerhof revised for solids (PBEsol) exchange correlation functional ${ }^{13}$ was used on a single $\Gamma$ point in the Brillouin-zone. Van der Waals corrections were applied using the DFTD3 method with Becke-Jonson damping. ${ }^{14-15}$ Optimizations proceeded via a three-step process. First, the ion positions were optimized with fixed cell dimensions, followed by an optimization of ions and cell parameters, and then ended with an additional ion-only optimization. A $400 \mathrm{eV}$ plane wave cutoff energy was used during ion optimizations, while a $500 \mathrm{eV}$ plane wave cutoff energy was used in the ion and cell optimization phase. Optimizations were continued until the force on all atoms was smallersd than $0.03 \mathrm{eV} \cdot \AA^{-1}$. Vibrational calculations were performed using the optimized structure with the same relevant input parameters.

As mentioned in the Main manuscript, IR vibrational frequencies and intensities were calculated by diagonalizing the Hessian matrix calculated using the density function perturbation theory (DFPT) method. ${ }^{16-19}$ Intensities within the DFPT framework are calculated via

$$
I(\omega)=\sum_{\alpha=1}^{3}\left|\sum_{s=1}^{M} \sum_{\beta=1}^{3} Z_{\alpha \beta}^{*}(s) e_{\beta}(s)\right|^{2}
$$

where $\alpha, \beta$ are Cartesian polarizations, $Z_{\alpha \beta}^{*}(s)$ is the Born effective charge of atom 's' , and $e_{\beta}(s)$ is the vibrational eigenvector of $\omega^{\text {th }}$ mode. A Lorentzian line shape, with a full width at half maximum of $20 \mathrm{~cm}^{-1}$, was applied for each frequency to simulate broadening effects. The calculated Lorentzian for all frequencies were summed together to form the full computational IR spectrum. Intensities were normalized such that the greatest intensity in the spectrum was unity. Identification of modes was accomplished by visualization within J-ICE. ${ }^{20}$ No scaling factor has 
been applied to calculated frequencies which leads to a general overprediction when compared to experiments. 


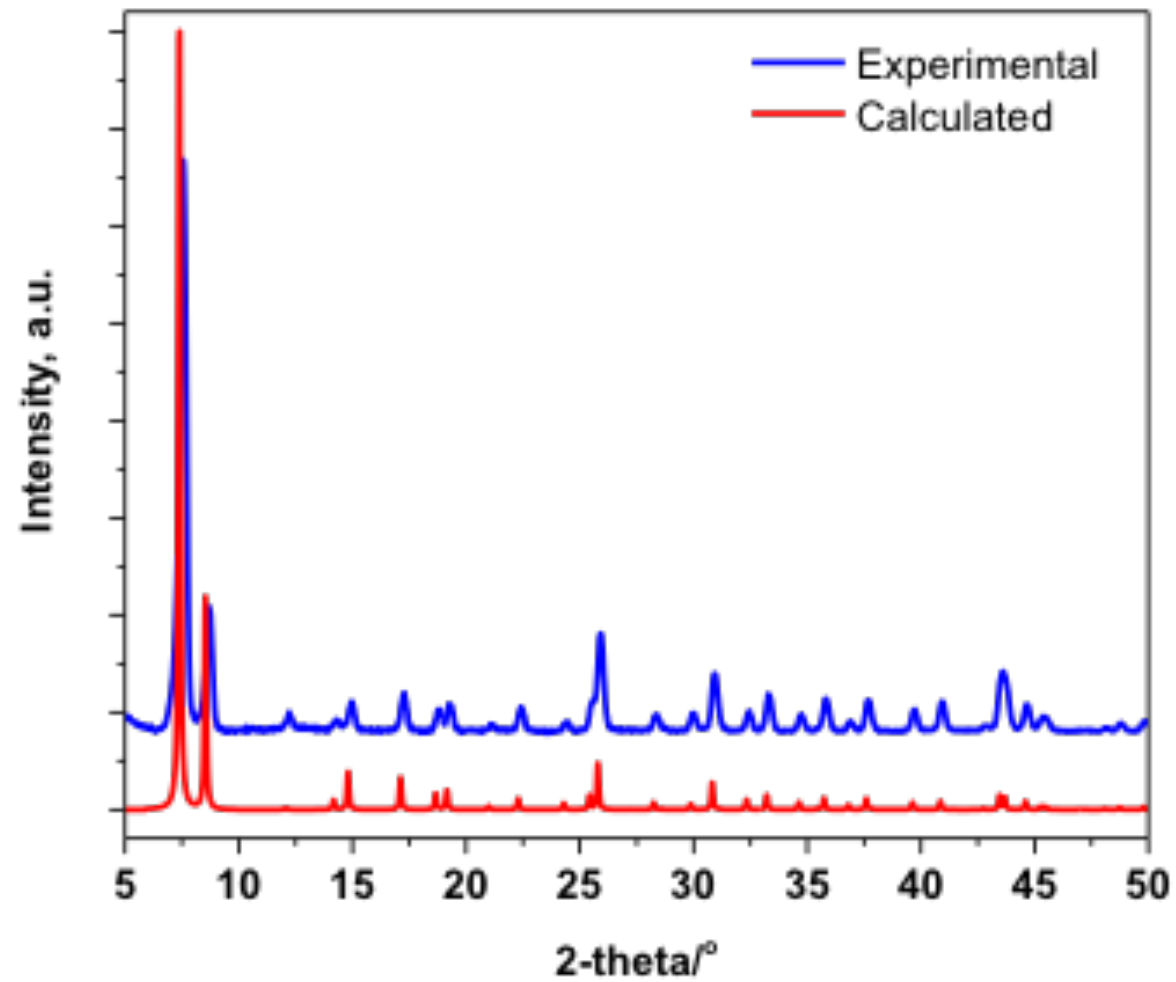

Figure S1. Powder X-ray diffraction patterns of experimental vs. calculated UiO-66 sample. 


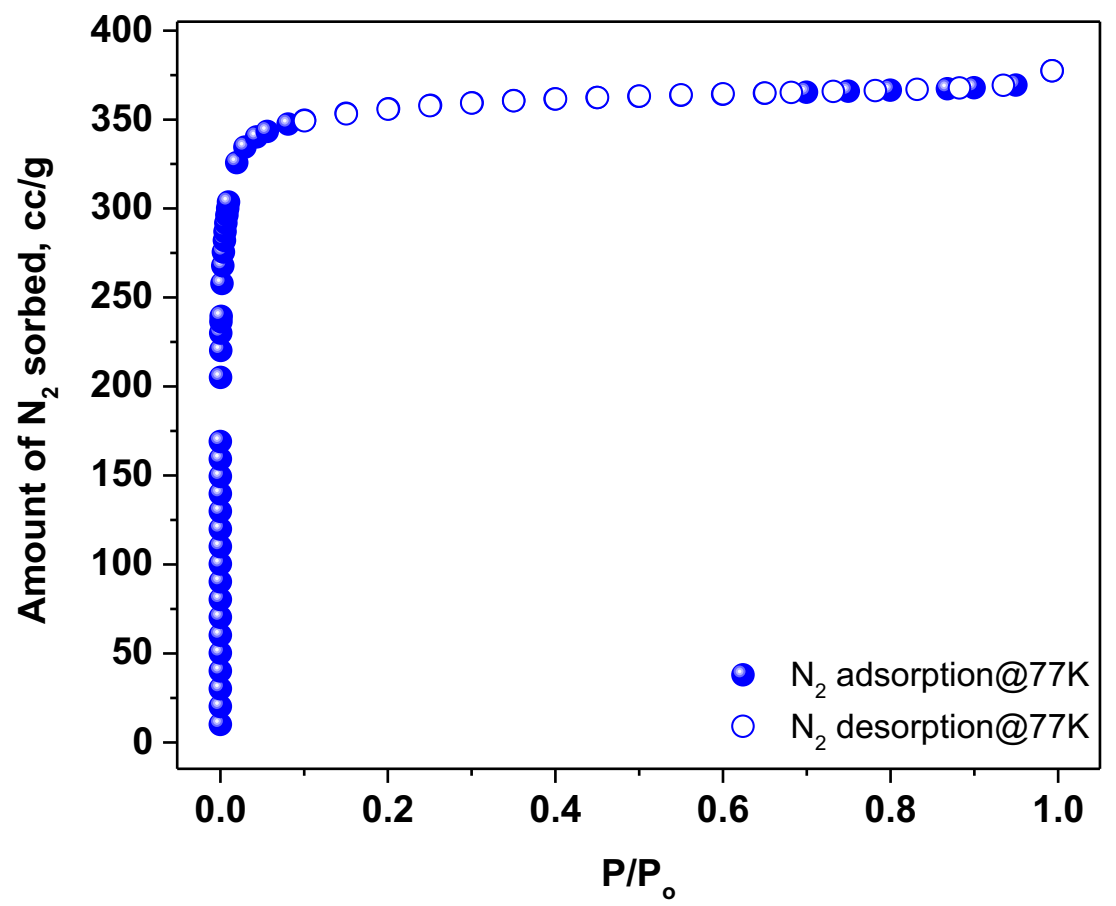

Figure S2. Nitrogen sorption isotherm measured at 77K on the desolvated UiO-66 sample. 


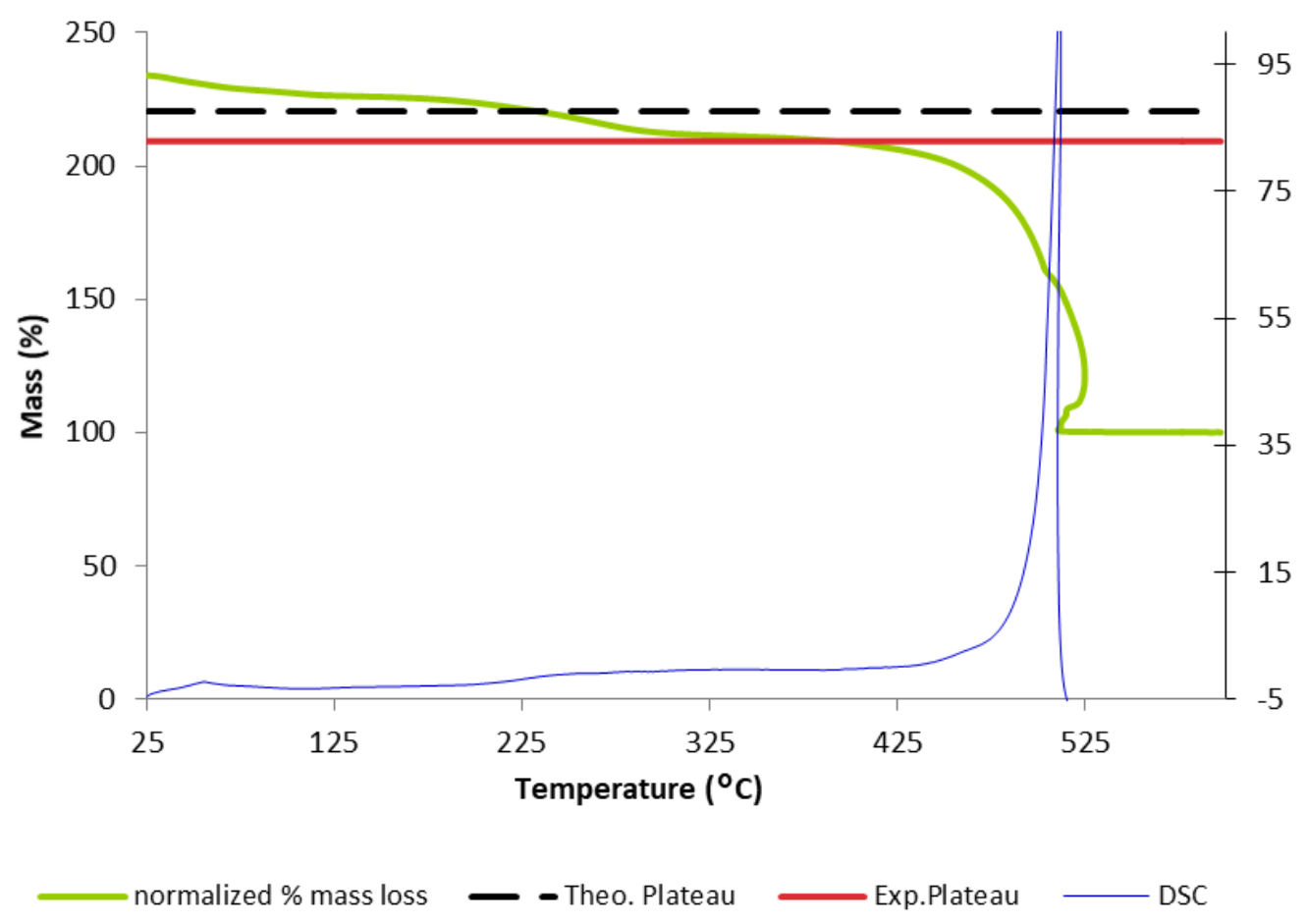

Figure S3. Thermogravimetric analysis of the UiO-66 sample. 


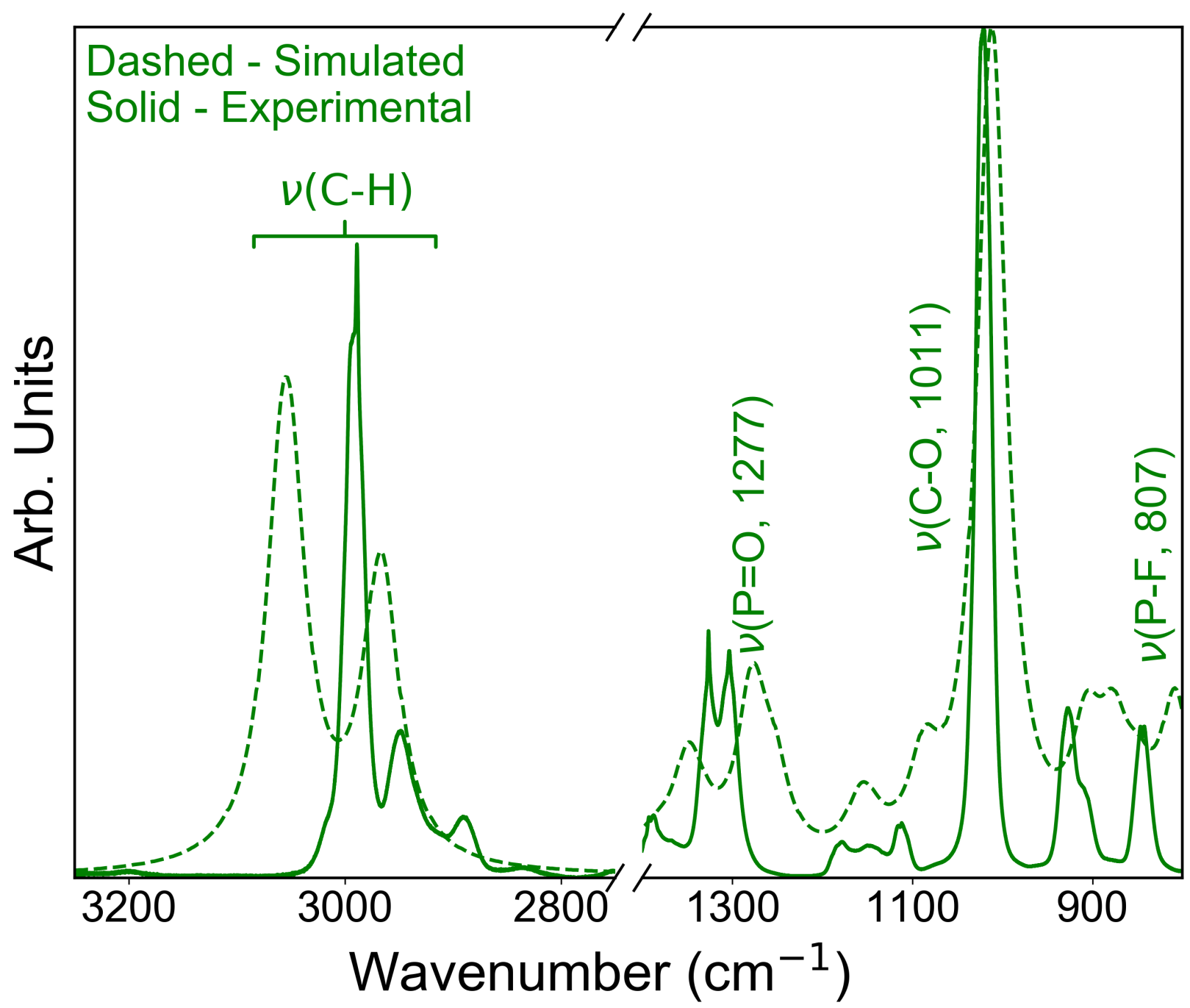

Figure S4. Simulated (dashed) and experimental (solid) IR spectrum for gas-phase GB. Intensities in the high-wavenumber region have been multiplied for visual clarity. The calculated frequency for the relevant $\mathrm{GB}$ modes including the $\mathrm{C}-\mathrm{H}, \mathrm{P}=\mathrm{O}, \mathrm{C}-\mathrm{O}$, and $\mathrm{P}-\mathrm{F}$ stretches are indicated. 


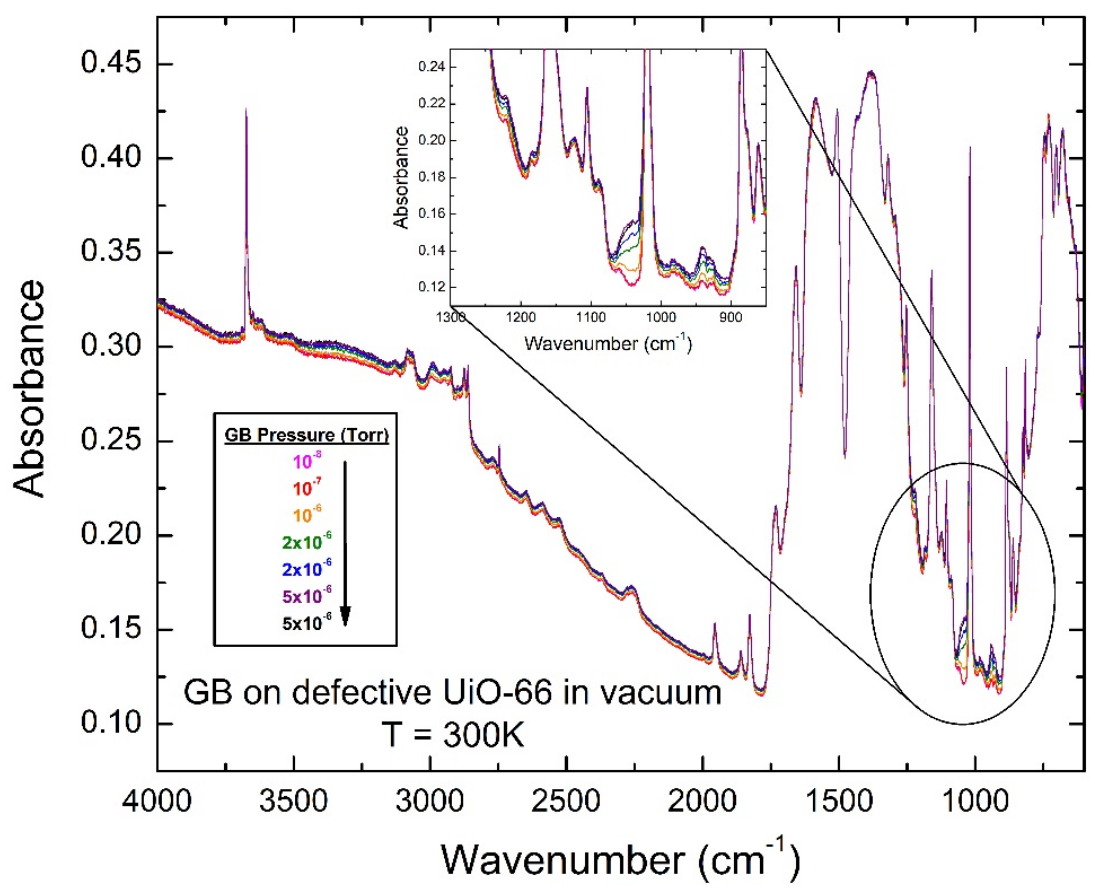

Figure S5. Raw absorbance spectra of GB exposure, with MOF modes included, at $10^{-8}$ (pink curve), $10^{-7}$ (red curve), $10^{-6}$ (orange curve), $2 \times 10^{-6}$ (green and blue curves), and $5 \times 10^{-6}$ (purple and black curves) Torr on UiO-66. The inset is a zoomed in IR region where the $\mathrm{P}=\mathrm{O}$ and $\mathrm{C}-\mathrm{O}$ moieties of GB are changing with GB pressure. 


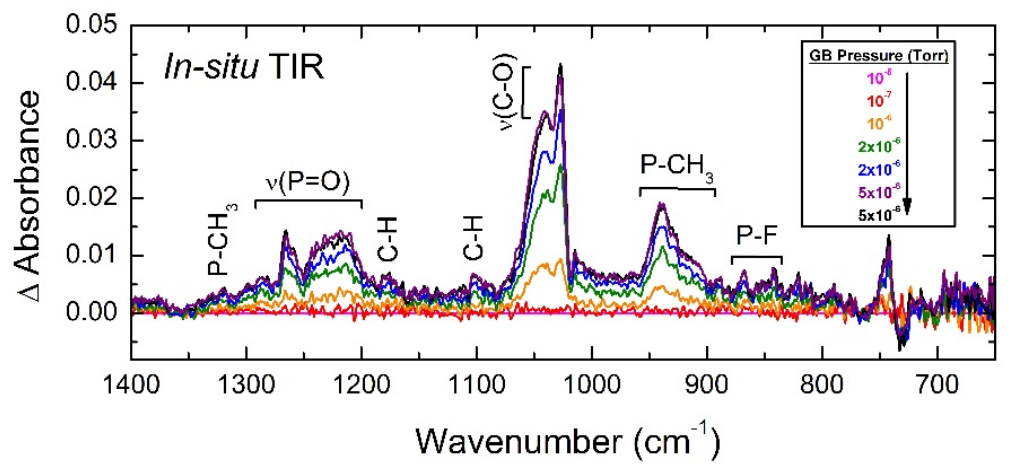

Figure S6. Difference spectra of GB exposure, calculated form Supplementary Information Figure S5, at $10^{-8}$ (pink curve), $10^{-7}$ (red curve), $10^{-6}$ (orange curve), $2 \times 10^{-6}$ (green and blue curves), and $5 \times 10^{-6}$ (purple and black curves) Torr on UiO-66. 
(a)

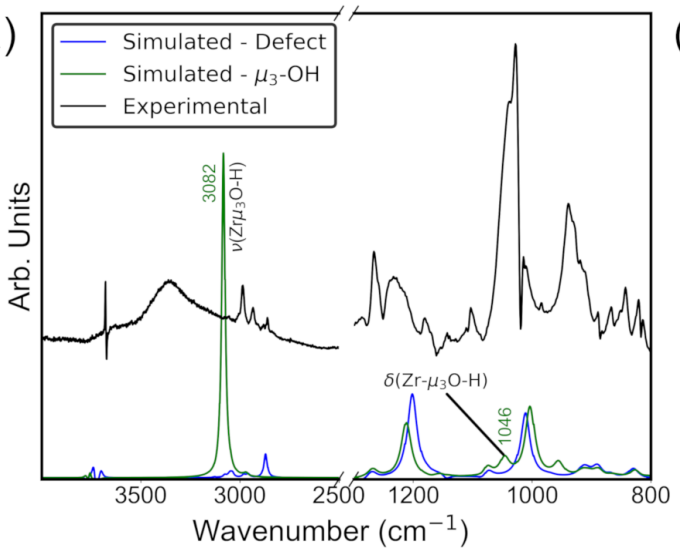

(b)

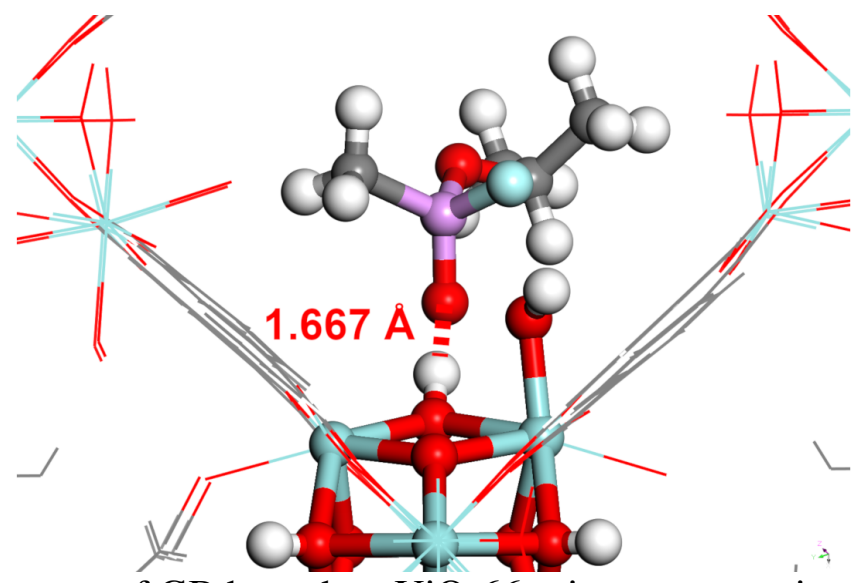

Figure S7. (a) Experimental (black) full IR spectrum of GB bound on UiO-66 prior to evacuation compared to simulated spectra for GB bound at a defect site (blue) and a $\mu_{3}-\mathrm{OH}$ site (green). Relevant modes for the $\mu_{3} \mathrm{O}-\mathrm{H}$ stretch $\left(3082 \mathrm{~cm}^{-1}\right)$ and bend $\left(1046 \mathrm{~cm}^{-1}\right)$ are indicated. (b) Structure of GB bound at the $\mu_{3}-\mathrm{OH}$ site with $\mathrm{P}=\mathrm{O} \cdots \mu_{3}-\mathrm{OH}$ distance indicated. 


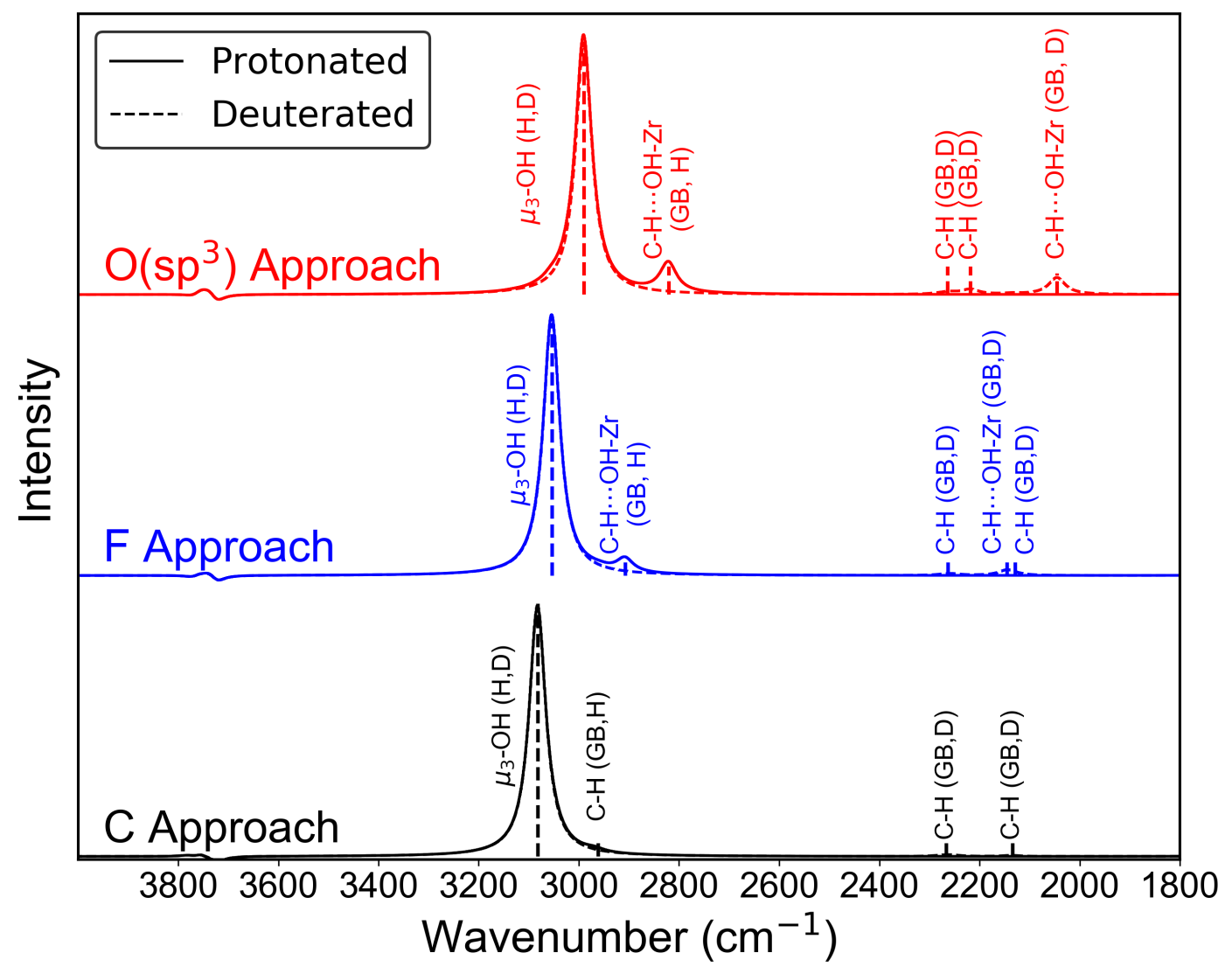

Figure S8. Simulated IR spectra for protonated (solid) and deuterated (dashed) GB bound at the $\mu_{3}-\mathrm{OH}$ site in the $\mathrm{O}\left(\mathrm{sp}^{3}\right)$ (red), F (blue), and C (black) orientations. See the manuscript text for description of orientations. 


\section{References}

(1) Shearer, G. C.; Chavan, S.; Ethiraj, J.; Vitillo, J. G.; Svelle, S.; Olsbye, U.; Lamberti, C.; Bordiga, S.; Lillerud, K. P. Tuned to Perfection: Ironing Out the Defects in Metal-Organic Framework UiO-66. Chem. Mater. 2014, 26, 4068-4071.

(2) Basu, P.; Ballinger, T. H.; Yates, J. T. Wide Temperature Range IR Spectroscopy Cell for Studies of Adsorption and Desorption on High Area Solids. Rev. Sci. Instrum. 1988, 59, 13211327.

(3) Cavka, J. H.; Jakobsen, S.; Olsbye, U.; Guillou, N.; Lamberti, C.; Bordiga, S.; Lillerud, K. P. A New Zirconium Inorganic Building Brick Forming Metal Organic Frameworks with Exceptional Stability. J. Am. Chem. Soc. 2008, 130, 13850-13851.

(4) Valenzano, L.; Civalleri, B.; Chavan, S.; Bordiga, S.; Nilsen, M. H.; Jakobsen, S.; Lillerud, K. P.; Lamberti, C. Disclosing the Complex Structure of UiO-66 Metal Organic Framework: A Synergic Combination of Experiment and Theory. Chem. Mater. 2011, 23, 1700-1718.

(5) Dassault Systèmes BIOVIA, Materials Studio, 2017 R2 (17.2 .0.1626), San Diego: Dassault Systèmes, 2017.

(6) Troya, D. Reaction Mechanism of Nerve-Agent Decomposition with Zr-Based Metal Organic Frameworks. J. Phys. Chem. C 2016, 120, 29312-29323.

(7) Blöchl, P. E. Projector Augmented-Wave Method. Phys. Rev. B 1994, 50, 17953-17979.

(8) Kresse, G.; Joubert, D. From Ultrasoft Pseudopotentials to the Projector Augmented-Wave Method. Phys. Rev. B 1999, 59, 1758-1775.

(9) Kresse, G.; Furthmuller, J. Efficiency of Ab-Initio Total Energy Calculations for Metals and Semiconductors Using a Plane-Wave Basis Set. Comp. Mater. Sci. 1996, 6, 15-50.

(10) Kresse, G.; Furthmuller, J. Efficient Iterative Schemes for Ab Initio Total-Energy Calculations Using a Plane-Wave Basis Set. Phys. Rev. B 1996, 54, 11169-11186.

(11) Kresse, G.; Hafner, J. Ab Initio Molecular-Dynamics Simulation of the Liquid-MetalAmorphous-Semiconductor Transition in Germanium. Phys. Rev. B 1994, 49, 14251-14269.

(12) Kresse, G.; Hafner, J. Ab Initio Molecular Dynamics for Liquid Metals. Phys. Rev. B 1993, 47, 558-561.

(13) Perdew, J. P.; Ruzsinszky, A.; Csonka, G. I.; Vydrov, O. A.; Scuseria, G. E.; Constantin, L. A.; Zhou, X.; Burke, K. Restoring the Density-Gradient Expansion for Exchange in Solids and Surfaces. Phys. Rev. Lett. 2008, 100, 136406.

(14) Grimme, S.; Ehrlich, S.; Goerigk, L. Effect of the Damping Function in Dispersion Corrected Density Functional Theory. J. Comput. Chem. 2011, 32, 1456-1465.

(15) Grimme, S.; Antony, J.; Ehrlich, S.; Krieg, H. A Consistent and Accurate Ab Initio Parametrization of Density Functional Dispersion Correction (DFT-D) for the 94 Elements H-Pu. J. Chem. Phys. 2010, 132, 154104.

(16) Baroni, S.; de Gironcoli, S.; Dal Corso, A.; Giannozzi, P. Phonons and Related Crystal Properties From Density-Functional Perturbation Theory. Rev. Mod. Phys. 2001, 73, 515-562.

(17) Giannozzi, P.; Baroni, S. Vibrational and Dielectric Properties of $\mathrm{C}_{60}$ From DensityFunctional Perturbation Theory. J. Chem. Phys. 1994, 100, 8537-8539.

(18) Giannozzi, P.; de Gironcoli, S.; Pavone, P.; Baroni, S. Ab Initio Calculation of Phonon Dispersions in Semiconductors. Phys. Rev. B 1991, 43, 7231-7242.

(19) Karhanek, D.; Bucko, T.; Hafner, J. A Density-Functional Study of the Adsorption of Methane-Thiol on the (111) Surfaces of the Ni-Group Metals: II. Vibrational Spectroscopy. $J$. Phys. Condens. Matter. 2010, 22, 265006. 
(20) Canepa, P.; Hanson, R. M.; Ugliengo, P.; Alfredsson, M. J-ICE: a New Jmol Interface for Handling and Visualizing Crystallographic and Electronic Properties. J. Appl. Crystallogr. 2011, $44,225-229$. 